\title{
The association between maternal body weight and vitamin $D$ status in early pregnancy: findings from the MO-VITD study
}

\author{
Raghad Alhomaid ${ }^{1}$, Maria Mulhern ${ }^{1}$, Laura Cassidy ${ }^{1}$, Eamon Laird ${ }^{2}$, Martin Healy ${ }^{3}$, \\ Sean Strain ${ }^{1}$, Barbara Livingstone ${ }^{1}$, Michael Parker ${ }^{4}$ and Mary McCann ${ }^{1}$ \\ ${ }^{1}$ Nutrition Innovation Centre for Food and Health, Ulster University, Londonderry, United Kingdom, \\ ${ }^{2}$ School of Biochemistry and Immunology, Trinity College Dublin, Dublin, Ireland, \\ ${ }^{3}$ Department of Biochemistry, Central Pathology Laboratory, St. James's Hospital, Dublin, Ireland and \\ ${ }^{4}$ Altnagelvin Area Hospital, Western Health and Social Care Trust, Londonderry, United Kingdom
}

\section{Abstract}

Maternal BMI has been shown to be inversely correlated with vitamin D status (25-hydroxyvitamin D (25(OH)D) concentrations) during pregnancy. Pregnant women with obesity and with vitamin D deficiency are at risk of many adverse health outcomes in pregnancy.

The aim of this study was to examine differences in maternal vitamin D status across normal weight, overweight and obese pregnant women in early pregnancy.

Data collected at baseline from a double-blind randomised vitamin D intervention study (MO-VITD) were used. Pregnant women without pregnancy complications, aged $>18$ years and having a singleton pregnancy were recruited between January 2016 and August 2017 at antenatal clinics in the Western Health and Social Care Trust, Northern Ireland. Non-fasting blood samples were collected at 12 weeks gestation and analysed for total serum $25(\mathrm{OH}) \mathrm{D}$, using liquid chromatography tandem mass spectrometry. Data from 239 pregnant women ( 80 normal weight, 79 overweight, 80 obese) were included in the current analysis.

The mean $\pm \mathrm{SD} 25(\mathrm{OH}) \mathrm{D}$ concentration of all pregnant women at 12 weeks gestation was $52.0 \pm 21.6 \mathrm{nmol} / \mathrm{L}$. Women classed as obese or overweight had significantly lower $25(\mathrm{OH}) \mathrm{D}$ concentrations compared to women of normal weight $(48.8 \pm 20.3 \mathrm{vs} 49.8 \pm 20.4$ vs. $57.5 \pm 23.1 \mathrm{nmol} / \mathrm{L}, P=0.019$; obese, overweight, normal weight respectively). A total of $45 \%$ of all pregnant women were found to be either vitamin $\mathrm{D}$ deficient $(25(\mathrm{OH}) \mathrm{D}<25 \mathrm{nmol} / \mathrm{L} ; 13 \%)$ or insufficient $(25-50 \mathrm{nmol} / \mathrm{L} ; 32 \%)$ in early pregnancy. BMI was significantly negatively correlated with $25(\mathrm{OH}) \mathrm{D}$ concentrations $(\boldsymbol{r}=-0.168 ; P=0.009)$. Regression analyses showed that BMI $(\beta=-0.165$; $P=0.006)$, season $(\beta=0.220 ; P=<0.0001)$, supplement use $(\beta=-0.268 ; P<0.0001)$ and a sun holiday within the previous 6 months $(\beta=-0.180 ; P=0.010)$ were significant predictors of $25(\mathrm{OH}) \mathrm{D}$ concentrations. In early pregnancy, $62 \%$ of pregnant women reported using a supplement containing vitamin D and $38 \%$ reported no supplement use. Supplement users had a significantly higher vitamin D status than non-supplement users in all BMI categories but overall, $37 \%$ of supplement users were still classified as vitamin D insufficient. Vitamin D status was significantly lower in winter months compared to summer months. In early pregnancy, especially during winter months, pregnant women with obesity, particularly non-supplement users, are at higher risk of low vitamin D status. Based on the lower vitamin D status observed in early pregnancy in obese women, the effect of BMI on vitamin D supplementation throughout pregnancy needs to be examined.

\section{Conflict of Interest}

There is no conflict of interest 